\title{
Low energy positron interactions with uracil-Total scattering, positronium formation, and differential elastic scattering cross sections
}

\author{
E. K. Anderson, ${ }^{1}$ R. A. Boadle, ${ }^{1}$ J. R. Machacek, ${ }^{1}$ L. Chiari, ${ }^{2}$ C. Makochekanwa, ${ }^{1}$ \\ S. J. Buckman, ${ }^{1,3, a)}$ M. J. Brunger, ${ }^{2,3}$ G. Garcia, ${ }^{4}$ F. Blanco, ${ }^{5}$ O. Ingolfsson, ${ }^{6}$ \\ and J. P. Sullivan ${ }^{1}$ \\ ${ }^{1}$ ARC Centre for Antimatter-Matter Studies, Research School of Physics and Engineering, \\ The Australian National University, Canberra 0200, Australia \\ ${ }^{2}$ ARC Centre for Antimatter-Matter Studies, Flinders University, GPO Box 2100, Adelaide, 5001 SA, Australia \\ ${ }^{3}$ Institute of Mathematical Sciences, University of Malaya, Kuala Lumpur, Malaysia \\ ${ }^{4}$ Instituto de Fisica Fundamental, Consejo Superior de Investigationes Cientıficas (CSIC), Serrano 113-bis, \\ E-28006 Madrid, Spain \\ ${ }^{5}$ Departamento de Fisica Atomica, Molecular y Nuclear, Universidad Complutense de Madrid, \\ E-28040 Madrid, Spain \\ ${ }^{6}$ Department of Chemistry, Science Institute, University of Iceland, Reykjavík 107, Iceland
}

(Received 1 April 2014; accepted 24 June 2014; published online 18 July 2014)

\begin{abstract}
Measurements of the grand total and total positronium formation cross sections for positron scattering from uracil have been performed for energies between 1 and $180 \mathrm{eV}$, using a trap-based beam apparatus. Angular, quasi-elastic differential cross section measurements at 1, 3, 5, 10, and $20 \mathrm{eV}$ are also presented and discussed. These measurements are compared to existing experimental results and theoretical calculations, including our own calculations using a variant of the independent atom approach. @ 2014 AIP Publishing LLC. [http://dx.doi.org/10.1063/1.4887072]
\end{abstract}

\section{INTRODUCTION}

Positrons, the anti-particle of the electron, are being used in an increasing number of contemporary applications in technology and medicine. These include their use in the nanoscale analysis of material structures, particularly those with open space or voids, the treatment of tumours (positherapy ${ }^{1}$ ), and medical imaging via positron emission tomography $\left(\mathrm{PET}^{2}\right)$. The latter imaging technology is now in widespread use in many major hospitals and is capable of the early detection of tumours, and other abnormalities, with a spatial resolution that can be as high as just a few mm. While this technology is well developed, little is understood of the overall transport of positrons in the body, from the moment of release at high energy from the radiopharmaceutical that is used (e.g., fluorodeoxyglucose or FDG), to the moment of annihilation, at typically very low energies, where the two back-to-back gamma rays, that are used for imaging, are produced. For ${ }^{18} \mathrm{~F}$, the isotope used in FDG, the mean energy of emission of the positrons is around $250 \mathrm{keV}$, and the energy distribution is very broad (also several hundred $\mathrm{keV}$ ) and they thermalize quickly (tens to hundreds of picoseconds) to sub-100 eV energies where the most dominant mechanism leading to annihilation, positronium formation, takes place with a high probability. The thermalisation process involves scattering and energy loss, through inelastic and ionization events with molecules in the body. It is establishing a quantitative knowledge of these processes that lies at the core of the present work. While studies of positron interactions with macroscopic biological entities such as proteins or cells are

\footnotetext{
a) Author to whom correspondence should be addressed. Electronic mail: Stephen.buckman@anu.edu.au.
}

not presently achievable, our aim is to combine quantitative, fundamental positron-molecule studies with state-of-the-art modeling techniques, in order to better understand positron transport and dosimetry. Such measurements also enable us to benchmark theoretical calculations for such molecules and assess the potential for such theories to be extended to more complex systems where experiments remain problematic. It is also unlikely that experiments on positron scattering or transport in real, soft condensed matter will be realized in the near future, so gas-phase measurements, such as those described here, together with modeling which includes structural factors of the medium, may be the key to such an advance. ${ }^{3,4}$ In that regard we have been engaged in a program of measurements of low energy positron interactions with biological molecules. To date these have included studies of water and formic acid, ${ }^{5,6}$ tetrahydrofuran, ${ }^{7}$ and pyrimidine. ${ }^{8}$

Uracil $\left(\mathrm{C}_{4} \mathrm{H}_{4} \mathrm{~N}_{2} \mathrm{O}_{2}\right)$ is an RNA nucleobase and, like the DNA nucleobases thymine and cytosine, it is a pyrimidine derivative. To our knowledge there has only been one previous measurement of positron interactions with uracil-a total scattering determination by the Wayne State University group ${ }^{9}$ and, also to the best of our knowledge, no ab initio scattering calculations exist for this molecule.

In this work we present new measurements for the scattering of positrons from the uracil molecule. These include total scattering, total positronium formation, and differential elastic scattering cross sections at energies between 1 and $180 \mathrm{eV}$. We also present calculated results for positron scattering from uracil using the independent atom model approach, including the screened additivity rule corrections (IAM-SCAR). In Sec. II we briefly discuss the experimental apparatus and techniques and the theoretical methods that have been applied. The results are presented and discussed, in 
comparison with previous work in Sec. III, and we conclude the paper with some final observations in Sec. IV.

\section{EXPERIMENTAL APPARATUS AND PROCEDURES AND THEORETICAL TECHNIQUES}

\section{A. Experimental approach}

The experimental apparatus has been described in complete detail in a number of recent papers ${ }^{10,11}$ so we shall only give a brief outline of the salient features here. It consists of a differentially pumped positron beamline with four main stages; the positron source, positron trap, scattering cell, and energy analyser/detector. Positrons from a $\sim 30 \mathrm{mCi}^{22} \mathrm{Na}$ source are moderated by a solid neon moderator, and guided by electric and magnetic fields to the trap stage, where they are trapped and accumulated through collisional cooling with $\mathrm{N}_{2}$ and $\mathrm{CF}_{4}$ gases. By carefully manipulating the electrostatic potentials on the last elements of the trap, the positrons are formed into a high resolution $(\sim 70 \mathrm{meV})$ pulsed beam, and directed to the scattering cell where they interact with the target molecules, before energy analysis using a retarding potential analyser, and detection with a channelplate detector.

Uracil $\left(\mathrm{C}_{4} \mathrm{H}_{4} \mathrm{~N}_{2} \mathrm{O}_{2}\right)$ is a white, solid powder at room temperature which has a melting point of $335^{\circ} \mathrm{C}$. In order to make a gaseous sample for the scattering measurements, controlled heating of the sample is required. To achieve this we have replaced our conventional scattering cell, as used for measurements on many room-temperature gases (e.g., Refs. 11-13), with a "hot cell" where a solid sample can be heated to provide sufficient vapour pressure for the scattering measurements. The heated scattering cell has similar dimensions to that used for our previous room-temperature gas experiments - it is a $100 \mathrm{~mm}$ long, $50 \mathrm{~mm}$ diameter, copper cylinder with $5 \mathrm{~mm}$ diameter apertures at either end. The cell is heated with twin-core Thermocoax resistive heating wire, which is wrapped in a helical fashion around the cylinder. The high thermal conductivity of copper, combined with the use of a stainless steel heat shield, generates a uniform temperature throughout the cell. A powder sample of the uracil target, typically around $0.3 \mathrm{mg}$, is loaded into a $1.4 \mathrm{~cm}^{3}$ crucible located at the base of the cell. The crucible can be removed to re-load the powder target without the need to disassemble the cell from the support structure. The crucible can also be heated independently to the cell with a separate Thermocoax wire, and it was typically held at a slightly higher temperature $\left(\sim 5^{\circ} \mathrm{C}\right)$ than the cell itself. The crucible and cell are heated to a temperature sufficient to sublime the sample and achieve a suitable vapour pressure for the scattering measurements. Typical operating temperatures for the present experiments, as measured with platinum resistance thermometers located on the cell and crucible, were $<130^{\circ} \mathrm{C}$.

In order to obtain the absolute scattering cross section the gas number density in the cell is required. However, for the present hot cell arrangement, the vapour pressure within the cell cannot be directly measured. This necessitated the use of vapour pressure curves for uracil from the literature in order to determine the pressure within the cell from the measured temperature. Vapour pressure studies of uracil by
Brunetti et al. ${ }^{14}$ show sufficient pressures of uracil should be obtained at temperatures below $\sim 130{ }^{\circ} \mathrm{C}$, which corresponds to a vapour pressure of $\sim 0.5$ mTorr. These authors also established an analytic expression for the vapour pressure as a function of temperature, and this was used to determine the cell pressure for all of the measurements in this work. This expression was derived for the temperature range $111^{\circ} \mathrm{C}-$ $221^{\circ} \mathrm{C}$, so the present work sits towards the bottom of this range.

One concern when creating such gas-phase molecular targets through thermal heating is the possibility for molecular dissociation to occur. In a series of experiments ${ }^{15}$ using a pulsed thermal source, uracil molecules were ionized by a frequency-tripled YAG laser, and the products were analysed in a time-of-flight mass spectrometer with unit mass resolution. ${ }^{16}$ These measurements demonstrated no evidence of dissociation of uracil at temperatures as high as $280^{\circ} \mathrm{C}$. As the typical operating temperatures in the present experiments were well below this value, we are confident that no dissociation of the uracil would have occurred.

Diffusion of target material out of the scattering cell is inevitable, since the scattering cell has openings at either end. While the scattering region is differentially pumped to minimise the diffusion of target material to other regions of the apparatus, particularly the trap and the moderator, this only works well for room-temperature gas targets. Condensable targets such as uracil can readily migrate and stick to surfaces, possibly causing contact potential shifts and surface charging effects. Our initial operation of the hot cell indicated significant deposition of uracil powder onto nearby surfaces of the vacuum chamber and scattering system that were at cooler temperatures. To minimise this potential contamination of the apparatus, two cold traps were added to the scattering region, one at either end of the scattering cell. Each cold trap consisted of a circular copper plate attached to a copper bar, which was in turn connected to a solid copper rod feedthrough in the vacuum chamber. The copper feedthrough was cooled externally by the use of a Peltier cell and, in this way, the copper plates and bars could be cooled to near $0^{\circ} \mathrm{C}$. The positron beam passed through a $6.5 \mathrm{~mm}$ diameter aperture in the centre of the cold plates and the plates proved effective in minimizing the effects of any condensed uracil on other areas of the apparatus.

The techniques used to obtain the total cross section, the positronium formation cross section, and the differential elastic scattering cross section have also been discussed in detail previously and we will not repeat that detail here, but refer the reader to those previous discussions (e.g., Refs. 11 and 17). Suffice it to say that with a knowledge of the transmitted positron intensities, the length of the scattering cell, and the gas vapour pressure, an absolute scattering cross section can be determined at any given positron energy.

One issue, which is of considerable importance for scattering-cell based measurements, is the consideration of forward elastic and inelastic scattering. We have discussed this issue at some length in several previous papers, ${ }^{12,18}$ and will not repeat the detailed rationale here. In summary, the failure to completely account for forward scattering leads to a reduction in the "measured" scattering cross section over the 
TABLE I. Estimated missing angular range $\left(\theta_{c}\right)$ (see text) for a range of energies within the present study. Also shown are the estimated missing part of the total cross section, expressed as a percentage, which are missing due to the restricted forward angle range. These estimates are based on the present theoretical calculation.

\begin{tabular}{lcc}
\hline \hline $\begin{array}{l}\text { Energy } \\
(\mathrm{eV})\end{array}$ & $\begin{array}{c}\theta_{c} \\
(\mathrm{deg})\end{array}$ & $\begin{array}{c}\text { Missing cross section } \\
(\% \text { of total })\end{array}$ \\
\hline 1 & 19 & 80 \\
2 & 14 & 75 \\
5 & 9 & 67 \\
10 & 6 & 55 \\
20 & 4.3 & 40 \\
50 & 2.7 & 26 \\
100 & 1.9 & 19 \\
\hline \hline
\end{tabular}

"true" value and, in cases where forward scattering is dominant due to the target possessing a permanent dipole moment and/or a large dipole polarizability, these effects can be significant. Uracil has both a large dipole moment $(\sim 3.8-$ 4.2 D, e.g., Ref. 19) and dipole polarisability (71.4 a.u. $\left.{ }^{20}\right)$, so a consideration of these forward scattering effects is likely to be critically important in a comparison between experiments, and experiment and theory.

In the present experimental arrangement, the angular range $\left( \pm \theta_{c}\right)$ in the forward direction over which scattering cannot be accounted for, is related to the energy resolution, and is energy dependent, being quite large at low energies and relatively insignificant at higher energies (see Table I). If we have knowledge of the differential scattering cross section (DCS), for example, from theory, we can estimate the extent that this angular discrimination effect has on the total cross section (TCS), and correct for it. This is discussed further in Sec. III.

The uncertainties on the present cross section measurements consist of contributions from statistical uncertainties, which are generally small, and contributions from the errors in the two central experimental parameters that determine the absolute cross section, the length of the scattering cell, and the absolute gas number density. The uncertainty in the former is known to be small $(<1 \%)$, while for the latter, the uncertainty may be considerable for the present experiment. The gas number density is proportional to the pressure in the gas cell $(\mathrm{P})$ which is related to the temperature of the cell $(\mathrm{T})$ and, according to Brunetti et al., ${ }^{14}$ it is given by the formula

$$
\log (\mathrm{P}(\mathrm{kPa}))=12.29( \pm 0.15)-6634( \pm 100) / \mathrm{T}(\mathrm{K}) .
$$

Applying this formula in the temperature region between $120^{\circ} \mathrm{C}$ and $130^{\circ} \mathrm{C}$ indicates that the uncertainties in the equation parameters lead to a possible uncertainty in the number density of more than $50 \%$. Furthermore, an uncertainty in the temperature measurement of $\pm 1^{\circ} \mathrm{C}$ (our estimate for the present experiment) would result in about a $10 \%$ uncertainty in the number density. Thus, while the statistical uncertainties on the measured cross sections, and the uncertainty on the energy dependence and angular distributions will be reasonably small ( $<\sim 10 \%)$, the absolute uncertainty on the present cross sections derived from the temperature measurement is difficult to define, but almost certainly larger than $50 \%$.
Another way that the experimental data can be put on an absolute scale is to normalize to a reliable theoretical calculation. In the present case we have done this using our independent atom model calculation, described below, at an energy of $150 \mathrm{eV}$. This calculation has been shown in previous applications for both electrons and positrons (e.g., Refs. 6 and 8 ) to provide a good description of the total scattering cross section at higher energies. Also, at these higher energies, the correction involved for the missing angle range is considerably smaller.

\section{B. Theoretical approach-The independent atom model}

Details of the application of the IAM-SCAR ${ }^{21}$ method to positron interactions have been provided in a number of previous papers (e.g., Refs. 7, 22, and 23), with the most relevant previous work being our measurements and calculation on water, ${ }^{6}$ so we will only very briefly summarise the approach here.

The local complex potential is given by

$$
V(r)=V_{\mathrm{S}}(r)+V_{\mathrm{P}}(r)+\mathrm{i} V_{\mathrm{A}}(r) .
$$

The real part of Eq. (2) drives the elastic scattering dynamics and embraces the electrostatic $\left(V_{\mathrm{S}}(r)\right)$ and polarization $\left(V_{\mathrm{P}}(r)\right)$ interactions. The imaginary part $\left(V_{\mathrm{A}}(r)\right)$ describes all the inelastic processes that are considered as absorption of flux from the incident positron beam. The static potential was obtained from the charge density derived from HartreeFock atomic wavefunctions. The dipole plus quadrupole polarization potential was developed from that reported by McEachran et al. ${ }^{24}$ and the absorption potential accounts for the electronic excitations, positronium (Ps) formation, and direct ionization. Representing the Ps formation channel is a challenging task and we have adopted a phenomenological approach, as discussed in Ref. 25.

To calculate the cross sections for positron collisions with uracil $\left(\mathrm{C}_{4} \mathrm{H}_{4} \mathrm{~N}_{2} \mathrm{O}_{2}\right)$, the additivity rule (AR) is then applied to the optical model results for each constituent atom. In this approach, the molecular scattering amplitude stems from the sum of all the relevant atomic amplitudes, including the phase coefficients, which gives the DCSs for the molecule of interest. ICSs can then be determined by integrating those DCSs, with the sum of the elastic and absorption ICSs (for all inelastic processes except rotations and vibrations) then giving the grand total cross sections (GTCS). The geometry of the molecule (atomic positions and bond lengths) is taken into account by using some screening coefficients and this, we believe, enables the range of validity of the technique to be extended down to impact energies of $\sim 30 \mathrm{eV}$ (or lower) for electron and positron scattering.

The IAM-SCAR approach described above does not account for vibrational and rotational excitations. However, for highly polar molecules such as uracil, additional dipoleinduced excitation cross sections can be calculated in the framework of the first Born approximation. These results can then be incorporated into our IAM-SCAR calculation in an incoherent way, just by adding up the cross sections as independent channels. The complete approach has been shown to be 
TABLE II. Grand total scattering cross sections for positron collisions with uracil (in units of $10^{-16} \mathrm{~cm}^{2}$ ). The left-hand column represents the asmeasured values using an absolute determination of the number density (see text). The right-hand column is the cross section that results from normalizing to the IAM calculation at an energy of $150 \mathrm{eV}$. The errors represent the estimated absolute uncertainty.

\begin{tabular}{|c|c|c|c|c|}
\hline $\begin{array}{l}\text { Energy } \\
(\mathrm{eV})\end{array}$ & $\begin{array}{l}\text { Total cross section } \\
\quad\left(10^{-16} \mathrm{~cm}^{2}\right)\end{array}$ & Error & $\begin{array}{l}\text { Scaled total cross section } \\
\qquad\left(10^{-16} \mathrm{~cm}^{2}\right)\end{array}$ & Error \\
\hline 1 & 44.1 & 25.6 & 97.4 & 56.5 \\
\hline 1.5 & 37.5 & 21.7 & 82.7 & 48.0 \\
\hline 2 & 33.5 & 19.4 & 73.9 & 42.9 \\
\hline 2.5 & 29.5 & 17.1 & 65.2 & 37.8 \\
\hline 3 & 27.0 & 15.6 & 59.5 & 34.5 \\
\hline 3.5 & 26.0 & 15.1 & 57.5 & 33.3 \\
\hline 4 & 24.4 & 14.2 & 53.9 & 31.2 \\
\hline 4.5 & 23.8 & 13.8 & 52.5 & 30.4 \\
\hline 5 & 22.9 & 13.3 & 50.5 & 29.3 \\
\hline 5.5 & 21.9 & 12.7 & 48.3 & 28.0 \\
\hline 6 & 21.3 & 12.4 & 47.1 & 27.3 \\
\hline 7 & 20.3 & 11.8 & 44.8 & 26.0 \\
\hline 8 & 19.2 & 11.2 & 42.5 & 24.6 \\
\hline 9 & 18.8 & 10.9 & 41.6 & 24.1 \\
\hline 10 & 18.5 & 10.7 & 40.8 & 23.7 \\
\hline 11 & 18.2 & 10.5 & 40.1 & 23.3 \\
\hline 12 & 17.8 & 10.3 & 39.3 & 22.8 \\
\hline 13 & 17.1 & 9.9 & 37.7 & 21.9 \\
\hline 14 & 17.5 & 10.2 & 38.7 & 22.4 \\
\hline 15 & 16.7 & 9.7 & 36.9 & 21.4 \\
\hline 16 & 16.8 & 9.7 & 37.1 & 21.5 \\
\hline 17 & 17.1 & 9.9 & 37.7 & 21.9 \\
\hline 18 & 15.6 & 9.1 & 34.5 & 20.0 \\
\hline 19 & 17.1 & 9.9 & 37.8 & 21.9 \\
\hline 20 & 15.4 & 8.9 & 34.0 & 19.7 \\
\hline 30 & 14.7 & 8.5 & 32.4 & 18.8 \\
\hline 40 & 14.5 & 8.4 & 31.9 & 18.5 \\
\hline 50 & 14.3 & 8.3 & 31.7 & 18.4 \\
\hline 60 & 13.4 & 7.8 & 29.5 & 17.1 \\
\hline 70 & 13.0 & 7.5 & 28.6 & 16.6 \\
\hline 80 & 13.1 & 7.6 & 28.9 & 16.8 \\
\hline 90 & 12.7 & 7.4 & 28.0 & 16.2 \\
\hline 100 & 12.7 & 7.4 & 28.1 & 16.3 \\
\hline 110 & 12.2 & 7.1 & 26.9 & 15.6 \\
\hline 120 & 11.9 & 6.9 & 26.3 & 15.3 \\
\hline 130 & 12.1 & 7.0 & 26.6 & 15.4 \\
\hline 140 & 11.7 & 6.8 & 25.8 & 15.0 \\
\hline 150 & 11.5 & 6.7 & 25.4 & 14.7 \\
\hline 160 & 11.4 & 6.6 & 25.1 & 14.6 \\
\hline 170 & 11.0 & 6.4 & 24.4 & 14.1 \\
\hline 180 & 10.6 & 6.1 & 23.3 & 13.5 \\
\hline
\end{tabular}

quite successful when applied to some polar molecules. . $^{6,8,26}$ In the present calculations a dipole moment of $3.87 \mathrm{D}$ was used. ${ }^{27}$

\section{RESULTS AND DISCUSSION}

The experimental total cross section, total positronium formation cross section, and elastic differential cross sections are presented in Tables II-IV, and illustrated in Figures 1-4. In Table II we provide two total cross section tabulations, one is the as-measured values using the vapour pressure estima-
TABLE III. The total positronium formation cross section for uracil (in units of $10^{-16} \mathrm{~cm}^{2}$ ). The column on the left is the as-measured experimental values, while the column on the right has been scaled (upwards) by a factor of 2.21 (see text). The errors represent the estimated absolute uncertainty.

\begin{tabular}{|c|c|c|c|c|}
\hline $\begin{array}{l}\text { Energy } \\
(\mathrm{eV})\end{array}$ & $\begin{array}{l}\text { Total Ps cross section } \\
\qquad\left(10^{-16} \mathrm{~cm}^{2}\right)\end{array}$ & Error & $\begin{array}{l}\text { Scaled total Ps cross section } \\
\qquad\left(10^{-16} \mathrm{~cm}^{2}\right)\end{array}$ & Error \\
\hline 1.0 & 0.84 & 0.49 & 1.86 & 1.08 \\
\hline 1.5 & 0.38 & 0.22 & 0.83 & 0.48 \\
\hline 2.0 & 0.56 & 0.33 & 1.24 & 0.72 \\
\hline 2.5 & 0.63 & 0.36 & 1.39 & 0.81 \\
\hline 3.0 & 1.35 & 0.78 & 2.98 & 1.73 \\
\hline 3.5 & 1.77 & 1.02 & 3.90 & 2.26 \\
\hline 4.0 & 2.50 & 1.45 & 5.52 & 3.20 \\
\hline 4.5 & 3.37 & 1.96 & 7.45 & 4.33 \\
\hline 5.0 & 3.71 & 2.15 & 8.20 & 4.76 \\
\hline 5.5 & 4.29 & 2.49 & 9.47 & 5.50 \\
\hline 6.0 & 4.08 & 2.36 & 9.00 & 5.23 \\
\hline 7.0 & 4.26 & 2.47 & 9.40 & 5.46 \\
\hline 8.0 & 3.99 & 2.31 & 8.80 & 5.11 \\
\hline 9.0 & 4.55 & 2.64 & 10.05 & 5.83 \\
\hline 10.0 & 4.62 & 2.68 & 10.21 & 5.93 \\
\hline 11.0 & 4.64 & 2.69 & 10.25 & 5.95 \\
\hline 12.0 & 4.98 & 2.89 & 10.99 & 6.38 \\
\hline 13.0 & 4.57 & 2.65 & 10.10 & 5.86 \\
\hline 14.0 & 4.97 & 2.88 & 10.96 & 6.36 \\
\hline 15.0 & 4.96 & 2.87 & 10.94 & 6.35 \\
\hline 16.0 & 4.56 & 2.65 & 10.07 & 5.85 \\
\hline 17.0 & 5.00 & 2.90 & 11.03 & 6.41 \\
\hline 18.0 & 4.82 & 2.79 & 10.64 & 6.18 \\
\hline 19.0 & 4.73 & 2.74 & 10.45 & 6.06 \\
\hline 20.0 & 4.69 & 2.72 & 10.36 & 6.01 \\
\hline 30.0 & 3.69 & 2.14 & 8.14 & 4.73 \\
\hline 40.0 & 3.09 & 1.79 & 6.82 & 3.96 \\
\hline 50.0 & 2.77 & 1.60 & 6.11 & 3.55 \\
\hline 60.0 & 2.48 & 1.44 & 5.48 & 3.18 \\
\hline 70.0 & 2.04 & 1.19 & 4.51 & 2.62 \\
\hline 80.0 & 1.76 & 1.02 & 3.89 & 2.26 \\
\hline 90.0 & 1.75 & 1.01 & 3.86 & 2.24 \\
\hline 100.0 & 1.70 & 0.98 & 3.75 & 2.17 \\
\hline 110.0 & 1.53 & 0.89 & 3.39 & 1.97 \\
\hline 120.0 & 1.50 & 0.87 & 3.32 & 1.93 \\
\hline 130.0 & 1.34 & 0.78 & 2.95 & 1.71 \\
\hline 140.0 & 1.35 & 0.79 & 2.99 & 1.74 \\
\hline 150.0 & 1.14 & 0.66 & 2.52 & 1.46 \\
\hline 160.0 & 1.48 & 0.86 & 3.27 & 1.90 \\
\hline 170.0 & 1.18 & 0.68 & 2.60 & 1.51 \\
\hline 180.0 & 1.21 & 0.70 & 2.66 & 1.55 \\
\hline
\end{tabular}

tion from the measured cell temperature, and the second is a cross section obtained by normalizing to the present theoretical calculation at an energy of $150 \mathrm{eV}$. Note that in this case we "adjusted" the theory, at each energy, to take into account the forward scattering range of the experiment in order to make the comparison more realistic (see Table I). The rationale for this normalization approach is discussed below.

In Figure 1 we compare the measured total cross section at energies between 1 and $180 \mathrm{eV}$ with the earlier measurement of Surdutovich et al. ${ }^{9}$ and with the present IAM calculation (note the log scale on the y-axis). The agreement between the present cross section and that of Surdutovich et al. 
TABLE IV. Elastic differential cross sections for positron scattering from uracil (in units of $10^{-16} \mathrm{~cm}^{2} / \mathrm{sr}$ ) at energies of (a) $1 \mathrm{eV}$; (b) $3 \mathrm{eV}$; (c) $5 \mathrm{eV}$; (d) $10 \mathrm{eV}$; (e) $20 \mathrm{eV}$. The errors indicated are statistical only.

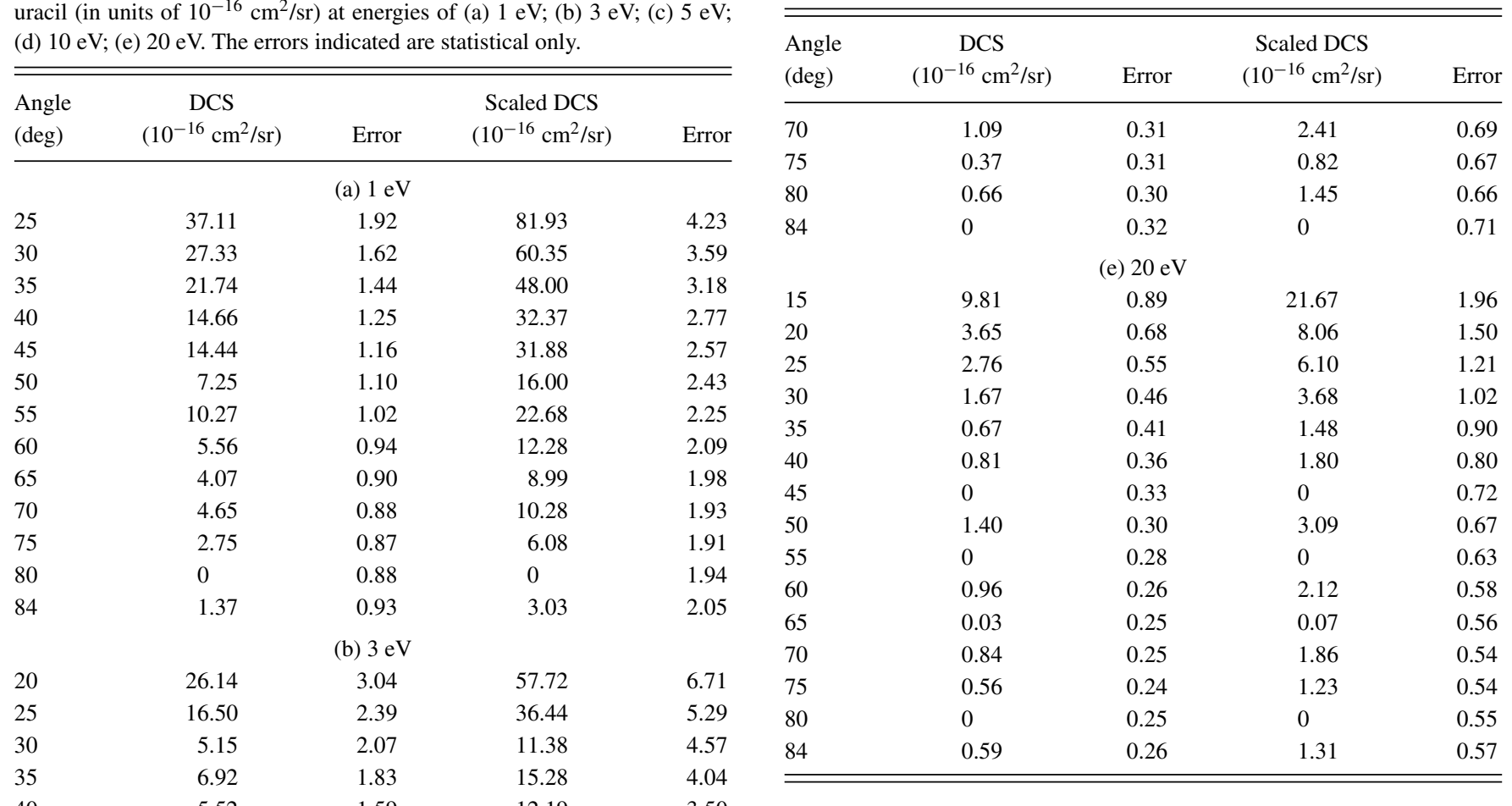

TABLE IV. (Continued.)

5.52

2.10

2.21

5.12

1.32

3.13

1.02

1.43

1.64

0.30

31.52

17.70

10.58

5.10

4.30

4.03

1.62

1.78

2.79

3.24

1.55

0.81

1.43

0.35

1.05

19.26

6.55

4.82

1.60

2.56

3.12

1.35

0.76

1.20

1.34

0.18

(c) $5 \mathrm{eV}$

1.66

1.27

1.02

0.86

0.77

0.71

0.61

0.58

0.55

0.52

0.49

0.48

0.46

0.46

0.49

69.60

3.67

2.79

2.25

1.90

1.70

1.56

1.35

1.28

1.21

1.14

1.07

1.05

1.02

1.01

1.09

(d) $10 \mathrm{eV}$

1.11

0.85

0.70

0.58

0.51

0.45

0.42

0.39

0.35

0.33

0.32

2.46

1.88

1.54

1.28

1.13

0.99

0.92

0.85

0.77

0.73

0.71

is reasonable, with the earlier measurement lying some $20 \%$ below the present across the common energy range. Both experiments used the same techniques and vapour pressure values to obtain the total cross section. It is likely that the difference between them could be entirely due to a larger missing angular range in the work of Ref. 9. It is also apparent in Figure 1 that both experiments lie well below the cross section calculated using the IAM approach. This is the case even at the highest energy of comparison, $180 \mathrm{eV}$, where the theory is still a factor of three higher than experiment.

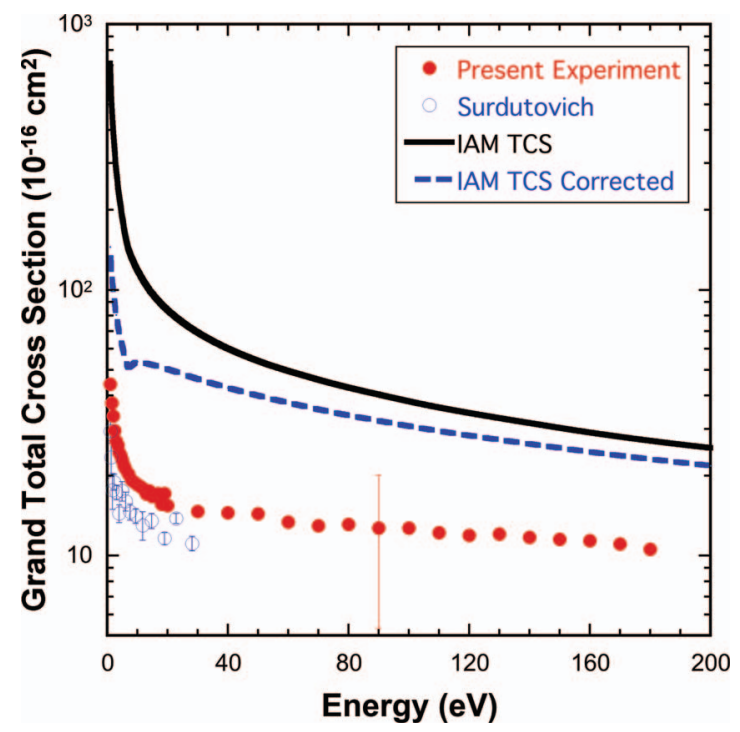

FIG. 1. The grand total scattering cross section (in units of $10^{-16} \mathrm{~cm}^{2}$ ) for positrons interacting with uracil molecules. The various symbols are explained in the plot legend. The small error bars on each point indicate the statistical uncertainty. The large error bar, at an energy of $90 \mathrm{eV}$, is indicative of the absolute uncertainty which results from the use of the uracil vapour pressure determination. 
Even when forward scattering effects in the experiment are factored in, using the values given in Table I, and the differential elastic and rotational cross sections from the theory, and the theoretical cross section is adjusted down to account for this, the adjusted theoretical cross section still lies well above the experiment at all energies.

This is somewhat puzzling, for several reasons. Recent work, ${ }^{6,8,26}$ some of it from our own collaboration, has demonstrated that the IAM approach can provide a good description of total and elastic differential cross sections for both electrons and positrons at energies above $30 \mathrm{eV}$ and, in some cases, even at lower energies. A significant case in point is the recent measurements for positron interactions with pyrimidine ${ }^{8}$ where we saw excellent agreement between the experiment and IAM theory across a broad energy range, particularly when the forward scattering issue was taken into account. Furthermore, the IAM calculation predicts similar magnitudes for the total cross sections for pyrimidine and uracil, with the uracil values being slightly larger, again as might be intuitively expected given their relative dipole moments and dipole polarizabilities (see later). On the other hand, the present experimental measurements for uracil indicate a total cross section that is between a factor of 2 and 4 smaller than that measured in our laboratory for pyrimidine, depending on the energy.

As a result of these arguments, we are led to believe that the most likely explanation for this significant discrepancy is the accuracy of the vapour pressure data for uracil, which was used in both the present experiment, and the Wayne State measurements, to determine the gas number density. On the other hand, the pyrimidine measurements in our laboratory ${ }^{8}$ were done by measuring the gas pressure directly. Pyrimidine is a relatively volatile liquid at room temperature and, for the range of pressures used in our experiments, the pressure could be accurately determined using a capacitance manometer. Thus, while we present the as-measured cross sections for uracil in Table II (left column) we also present tabulated cross section values in the right column obtained through a straightforward normalisation to the (forward-angle adjusted) IAM theory at $150 \mathrm{eV}$. Note that although the missing angle in the experiment is smaller at energies above $100 \mathrm{eV}$, a correction is still needed due to the very high rotational excitation cross section which dominates at small angles. The normalized cross section is shown in comparison with the adjusted IAM calculation in Figure 2. The agreement at high and low energies is rather good, while between 10 and $100 \mathrm{eV}$ the angle-adjusted theory is still significantly higher than experiment.

The total cross section for positronium (Ps) formation is given in Table III and shown in Figure 3, where it is compared with the phenomenological IAM estimate of the Ps cross section. The only real point of agreement between the experiment and theory would appear to be the position of the maximum of the Ps formation cross section at around $10 \mathrm{eV}-$ about $8 \mathrm{eV}$ above the Ps formation threshold at $2.54 \mathrm{eV}$. The experiment shows a steep rise in the cross section up to $5 \mathrm{eV}$ and then a rather flat maximum region, with a magnitude near $5 \AA^{2}$ that extends to around $20 \mathrm{eV}$, before a gradual decline to a value of around $1 \AA^{2}$ at $180 \mathrm{eV}$. We note that this peak value of the

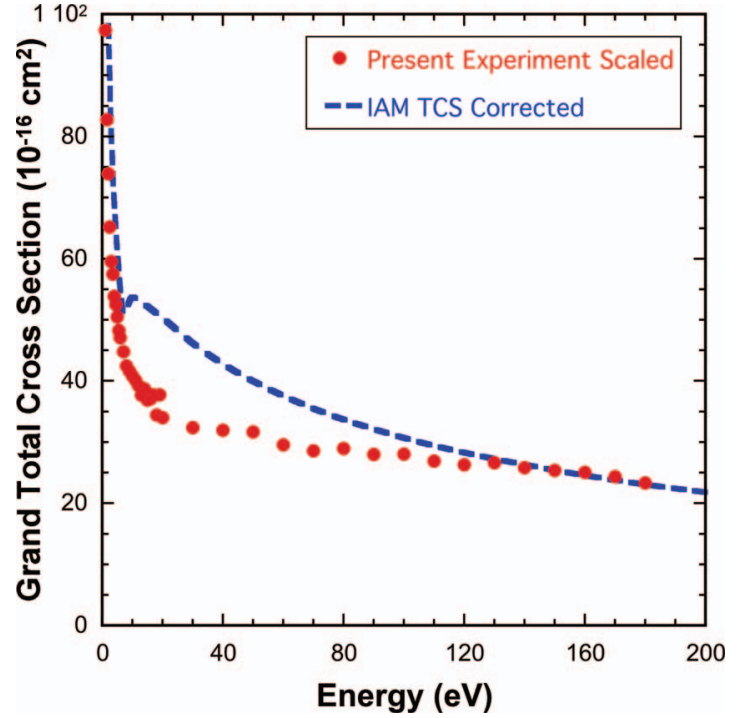

FIG. 2. The scaled grand total scattering cross section (in units of $10^{-16} \mathrm{~cm}^{2}$ ) for uracil (see text) compared to the adjusted IAM calculation. The various symbols are explained in the plot legend.

Ps cross section for uracil is about a factor of three smaller than that observed for pyrimidine when, intuitively, we might expect it to be somewhat larger than pyrimidine, given the relative sizes of the molecules, amongst other factors (discussed below). This further supports the argument above that there is a problem with the vapour pressure determination from the cell temperature measurement. If we scale the Ps formation cross section by the same normalisation factor used for the total cross section (2.21), the resulting peak cross section is in much better agreement with the IAM estimate (also shown in Figure 3). The other point to note about the Ps cross section

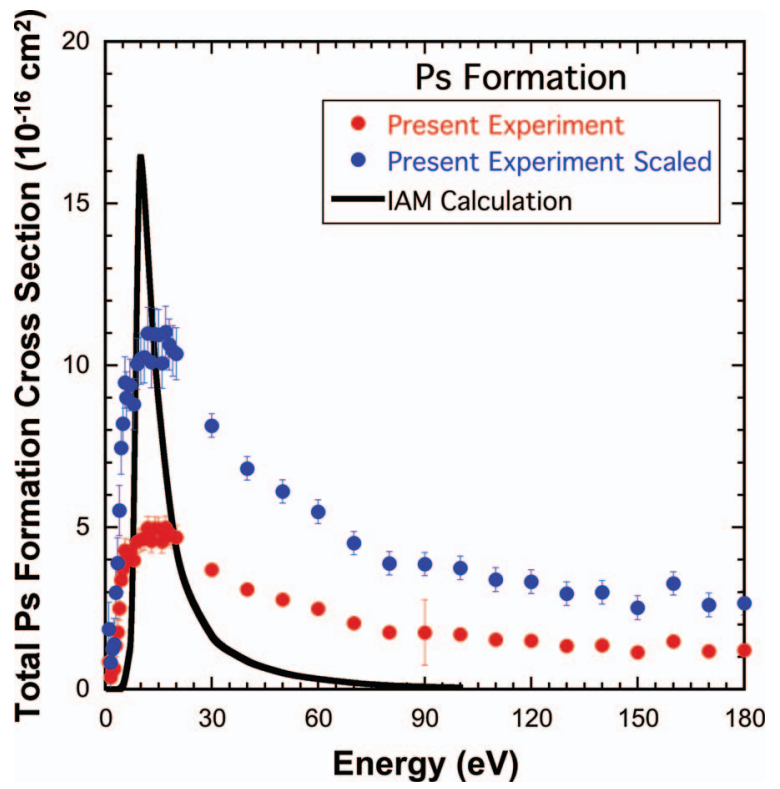

FIG. 3. The total Ps formation cross section (in units of $10^{-16} \mathrm{~cm}^{2}$ ) compared with the IAM calculation. The scaled Ps cross section has been multiplied by a factor of 2.21. The various symbols are explained in the plot legend. The small error bars on each point indicate the statistical uncertainty. The large error bar, at an energy of $90 \mathrm{eV}$, is indicative of the absolute uncertainty which results from the use of the uracil vapour pressure determination. 
for uracil is that it is significantly larger at high energies (even before scaling) than we have observed in other targets where, by $180 \mathrm{eV}$ the Ps cross section has normally been approaching zero.

We have also measured quasi-elastic differential cross sections at a range of energies between 1 and $20 \mathrm{eV}$. In this context the measurements are "quasi-elastic" as we cannot
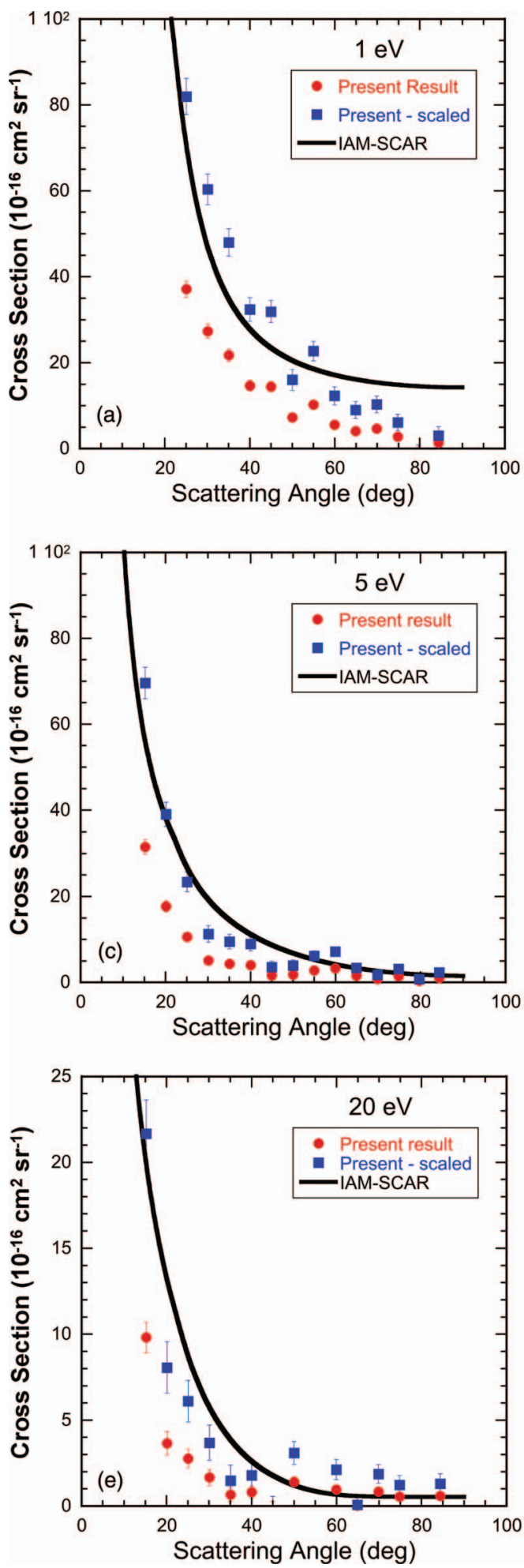
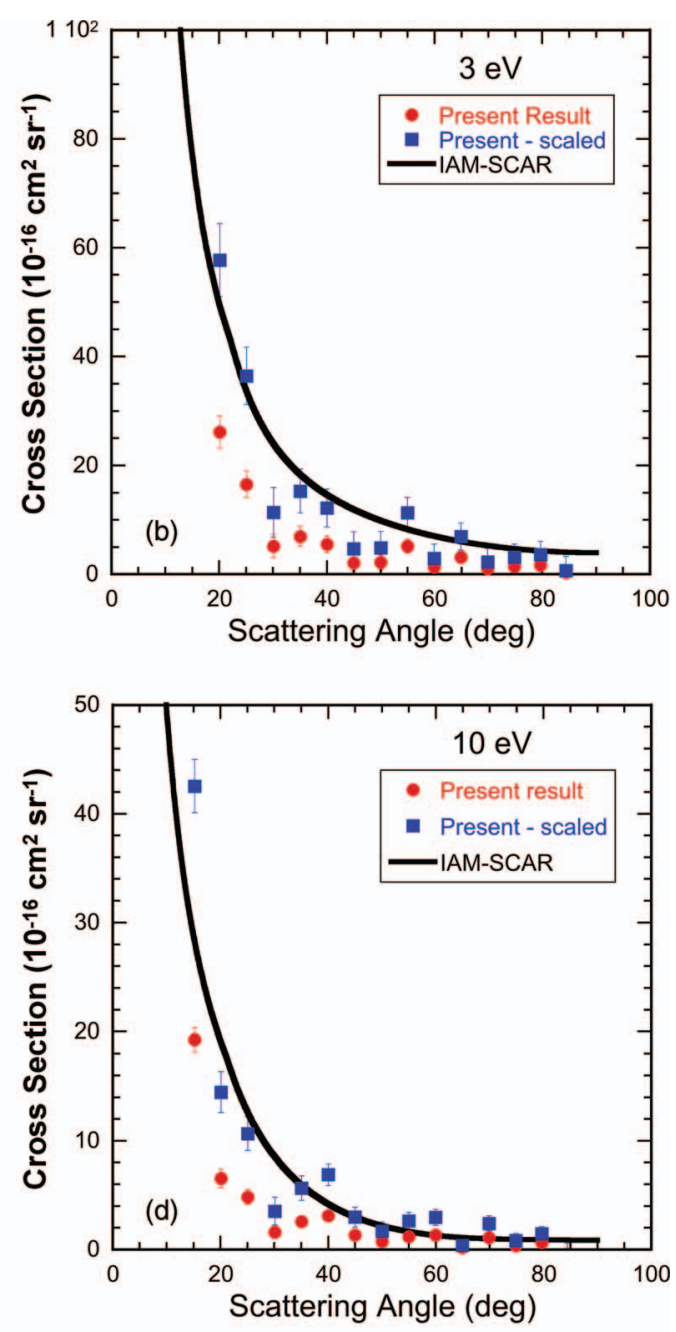

resolve rotational and some vibrational contributions from the purely elastic scattering with the present energy resolution (this has been discussed in detail previously, 6,28 ). Tabulated values of these as-measured cross sections, together with values which have been adjusted with the IAM normalization factor of 2.21 , are given in Table IV, and the results are shown graphically in Figures 4(a)-4(e). The rationale for

FIG. 4. Elastic differential cross sections for positrons scattered from uracil at (a) $1 \mathrm{eV}$, (b) $3 \mathrm{eV}$, (c) $5 \mathrm{eV}$, (d) $10 \mathrm{eV}$, and (e) $20 \mathrm{eV}$ (in units of $10^{-16} \mathrm{~cm}^{2} / \mathrm{sr}$. The scaled cross sections have been multiplied by a factor of 2.21. The various symbols are explained in the plot legend. The error bars that are indicated are the statistical uncertainties only. 
these scaled values is that any uncertainty in the gas number density determination, as speculated above, will also affect the magnitude of the measured differential cross sections, but not the angular distribution.

The DCS in Figures 4(a)-4(e) all show the same general trends, and similar trends that have been observed in differential positron scattering for a range of other polar molecules. ${ }^{6,8}$ Namely, the DCS are substantially peaked in the forward direction, and generally largest at low incident energies and angles-all traits that are common to both positron and electron scattering from polar (and highly polarizable) targets such as uracil, pyrimidine, and water. It is readily observed that, at all energies, the as-measured experimental DCS lie well below the IAM calculation. While at these low energies we may not a priori expect the IAM calculation for the elastic DCS to be particularly accurate, we have in the past observed a good level of accord between measurements of the elastic DCS for both water and pyrimidine, and the calculated IAM DCS. Thus, the large discrepancies between experiment and theory for uracil would appear to be inconsistent with previous comparisons for other polar and large molecular systems.

When we compare the scaled DCS values with the IAM calculation, we see considerably better agreement at all energies and this, perhaps, lends further weight to the notion that there is an issue with the absolute number density calculations based on the vapour pressure data of reference, ${ }^{14}$ or some other, unknown, issue.

\section{CONCLUSIONS}

We have measured absolute cross sections for total scattering, positronium formation, and elastic differential scattering for low to intermediate energy positrons interacting with uracil molecules. The level of agreement between the present, as-measured total cross sections and the one previous measurement ${ }^{9}$ is reasonably good, but both lie well below the present theoretical calculation, even at high energies where one might expect theory and experiment to converge. This, together with a comparison between the present data and previous measurements for the pyrimidine molecule, indicates the present data may be anomalously low, by a factor of 2-3. The normalisation of the present total cross section to the present IAM calculation, at an energy of $150 \mathrm{eV}$, indicates a scaling factor (upwards) of 2.21 for the present data. If one was to (somewhat arbitrarily) require the present peak magnitude of the Ps formation cross section for uracil to be equivalent to that measured for pyrimidine, then we would need to scale the present data upwards by a factor of 2.42 . Note that while this latter scaling is somewhat arbitrary, one might actually expect that the peak Ps cross section for uracil should be larger than that for pyrimidine on the basis of simple considerations of the number of electrons in each molecule and the molecular structure. Uracil has 58 electrons, a dipole moment of $\sim 3.8$ $4.2 \mathrm{D}$, and dipole polarisability of $\sim 71$ a.u., while pyrimidine possesses 42 electrons and has a dipole moment of $\sim 2.3 \mathrm{D}$ and a dipole polarisability of $\sim 59$ a.u.

The above considerations all led us to speculate that the temperature vs vapour pressure conversion factors that were used in the present experiment, and in that of Surdutovich et al., may be in error, and that the as-measured values of all the cross sections in the present work need to be scaled upwards by a factor somewhere between 2 and 2.5.

When this scaling is done, the agreement between experiment and theory is more reasonable, as might be anticipated following the earlier successes observed with both water and pyrimidine. The approach used in the IAM calculation to obtain a phenomenological estimate of the Ps formation cross sections seems to provide a good level of agreement with the peak value of the (scaled) experimental cross section, but the energy dependence, at higher energies, does not match experiment, with the theory falling away much too quickly with energy. Once again, the surprise in the comparison with theory was the good level of agreement between theory and (scaled) experiment for the elastic DCS, as was also observed in water and pyrimidine. It is also surprising that this good agreement should extend to such low energies, but indicates that the IAM method may be a very useful technique for readily generating cross sections for positron interactions with large, experimentally inaccessible molecules.

In our past studies on tetrahydrofuran $\left(\mathrm{C}_{4} \mathrm{H}_{8} \mathrm{O}\right)$, 3-hydroxy-tetrahydrofuran $\left(\mathrm{C}_{4} \mathrm{H}_{8} \mathrm{O}_{2}\right)$, and pyrimidine $\left(\mathrm{C}_{4} \mathrm{H}_{4} \mathrm{~N}_{2}\right)$, the measured positronium formation cross sections have essentially gone to zero at energies of around 150-180 eV. This is in stark contrast to uracil $\left(\mathrm{C}_{4} \mathrm{H}_{8} \mathrm{~N}_{2} \mathrm{O}_{2}\right)$, where the positronium formation cross section is non-zero up to our highest measured energy $(180 \mathrm{eV})$. Tetrahydrofuran has 40 electrons, pyrimidine has 42 electrons, 3-hydroxytetrahydrofuran has 48 electrons, while uracil possesses 58 electrons. Hence, the electronic-structure of uracil, in terms of both the valence and core electrons, is far richer than for each of the other 3 species. In addition, the electron cloud distribution for uracil is far more extended than that of tetrahydrofuran, pyrimidine, and 3-hydroxy-tetrahydrofuran. We believe this is the reason that the positronium formation cross section persists to higher energies in uracil than for the other 3 targets. The results of Figure 3 also clearly illustrate that, at higher energies in particular, the phenomenological theoretical approach that we have used is not successful in predicting the magnitude of Ps formation cross section. While this clearly represents a limitation in this phenomenological approach, we believe that the theoretical results are useful at a semi-quantitative level, and add to our understanding of the positron-uracil scattering dynamics.

We finally comment that the forward angle effects which we have outlined in detail in the text, clearly pose a challenge for comparison between experiment and theory at the total cross section level and, at low energies, they can be so large that the whole process becomes quite approximate. Nonetheless, we believe that the thoughtful combination of experiment and theory can still be used to give reasonable estimates of the total scattering cross section.

\section{ACKNOWLEDGMENTS}

This work was supported by the Australian Research Council through its Centre of Excellence Program. We also acknowledge the support of the Spanish Ministerio de Economia y Competitivad under Project No. FIS 2101-31230. The 
experimental program would not have been possible without the outstanding technical support provided by Ross Tranter and Stephen Battisson. O.I. gratefully acknowledges support by the CAMS Centre, the ANU and Flinders University, and by the University of Iceland during his sabbatical period in Australia. S.J.B. and M.J.B. are both grateful to the University of Malaya for their hospitality during periods as Distinguished Visiting Professors.

${ }^{1}$ L. Menichetti, L. Cionini, W. A. Sauerwein, S. Altieri, O. Solin, H. Minn, and P. Salvadori, Appl. Radiat. Isot. 67, S351 (2009).

${ }^{2}$ D. Bailey, W. Townsend, P. Valk, and M. Maisey, Positron Emission Tomography (Springer-Verlag, London, 2005).

${ }^{3}$ R. D. White, W. Tattersall, G. Boyle, R. E. Robson, S. Dujko, Z. Lj. Petrovic, A. Bankovic, M. J. Brunger, J. P. Sullivan, S. J. Buckman, and G. Garcia, Appl. Radiat. Isotop. 83B, 77 (2014).

${ }^{4}$ Z. Lj. Petrovic, S. Marjanovic, S. Dujko, A. Bankovic, G. Malovic, S. J. Buckman, G. Garcia, R. White, and M. J. Brunger, Appl. Radiat. Isot. 83B, 148 (2014).

${ }^{5}$ C. Makochekanwa, A. Bankovic, W. Tattersall, A. Jones, P. Caradonna, D. Slaughter, J. P. Sullivan, K. Nixon, M. J. Brunger, Z. Lj. Petrovic, and S. J. Buckman, New J. Phys. 11, 103036 (2009).

${ }^{6}$ W. Tattersall, L. Chiari, J. R. Machacek, E. Anderson, R. D. White, M. J. Brunger, S. J. Buckman, G. Garcia, F. Blanco, and J. P. Sullivan, J. Chem. Phys. 140, 044320 (2014).

${ }^{7}$ L. Chiari, E. Anderson, W. Tattersall, J. Machacek, P. Palihawadana, C. Makochekanwa, J. P. Sullivan, G. García, F. Blanco, R. P. McEachran, M. J. Brunger, and S. J. Buckman, J. Chem. Phys. 138, 074301 (2013).

${ }^{8}$ P. Palihawadana, R. Boadle, L. Chiari, E. K. Anderson, J. Machacek, M. J. Brunger, S. J. Buckman, and J. P. Sullivan, Phys. Rev. A 88, 012717 (2013).

${ }^{9}$ E. Surdutovich, G. Setzler, W. E. Kauppila, S. J. Rehse, and T. S. Stein, Phys. Rev. A 77, 054701 (2008).

${ }^{10}$ J. P. Sullivan, A. Jones, P. Caradonna, C. Makochekanwa, and S. J. Buckman, Rev. Sci. Instrum. 79, 113105 (2008).

${ }^{11}$ J. R. Machacek, C. Makochekanwa, A. Jones, P. Caradonna, D. Slaughter, R. P. McEachran, J. P. Sullivan, S. J. Buckman, S. Bellm, B. Lohmann, D.
Fursa, I. Bray, D. W. Mueller, and A. D. Stauffer, New J. Phys. 13, 125004 (2011).

${ }^{12}$ A. Jones, C. Makochekanwa, P. Caradonna, D. Slaughter, J. Machacek, R. P. McEachran, J. P. Sullivan, S. J. Buckman, A. D. Stauffer, I. Bray, and D. Fursa, Phys. Rev. A 83, 032701 (2011).

${ }^{13}$ C. Makochekanwa, J. Machacek, A. Jones, P. Caradonna, D. Slaughter, R. P. McEachran, J. P. Sullivan, S. J. Buckman, S. Bellm, B. Lohmann, D. W. Mueller, A. D. Stauffer, D. Fursa, I. Bray, and M. Hoshino, Phys. Rev. A 83, 032721 (2011).

${ }^{14}$ B. Brunetti, V. Piacente, and G. Portalone, J. Chem. Eng. Data 45, 242 (2000).

${ }^{15}$ T. M. Maddern, private communication (2012).

${ }^{16}$ T. M. Maddern, L. R. Hargreaves, M. Bolarizadeh, M. J. Brunger, and S. J. Buckman, Meas. Sci. Technol. 19, 085801 (2008).

${ }^{17}$ J. P. Sullivan, S. J. Gilbert, J. P. Marler, R. G. Greaves, S. J. Buckman, and C. M. Surko, Phys. Rev. A 66, 042708 (2002).

${ }^{18}$ J. P. Sullivan, C. Makochekanwa, A. Jones, P. Caradonna, D. Slaughter, J. Machacek, R. P. McEachran, D. W. Mueller, and S. J. Buckman, J. Phys. B 44, 035201 (2011).

${ }^{19}$ I. Kulakowska, M. Geller, B. Lesyng, and K. L. Wierzchowski, Biochim. Biophys. Acta 361, 119 (1974).

${ }^{20}$ T. Pluta, M. Kolaski, M. Medved, and S. Budzák, Chem. Phys. Lett. 546, 24 (2009).

${ }^{21}$ F. Blanco and G. Garcia, J. Phys. B 42, 145203 (2009).

${ }^{22}$ L. Chiari, A. Zecca, S. Girardi, E. Trainotti, G. Garcia, F. Blanco, R. P. McEachran, and M. J. Brunger, J. Phys. B 45, 215206 (2012).

${ }^{23}$ L. Chiari, P. Palihawadana, J. Machacek, C. Makochekanwa, J. P. Sullivan, G. García, F. Blanco, R. P. McEachran, M. J. Brunger, and S. J. Buckman, J. Chem. Phys. 138, 074302 (2013).

${ }^{24}$ R. P. McEachran, D. L. Morgan, A. G. Ryman, and A. D. Stauffer, J. Phys. B 10, 663 (1977).

${ }^{25}$ L. Chiari, A. Zecca, E. Trainotti, G. Garcia, F. Blanco, M. H. F. Bettega, S. d'A Sanchez, M. T. Varella, M. A. P. Lima, and M. J. Brunger, Phys. Rev. A 88, 022708 (2013).

${ }^{26}$ L. Chiari, A. Zecca, G. Garcia, F. Blanco, and M. J. Brunger, J. Phys. B 46, 235202 (2013).

${ }^{27}$ R. D. Brown, P. D. Godfrey, D. McNaughton, and A. P. Pierlot, J. Am. Chem. Soc. 110, 2329 (1988).

${ }^{28}$ J. R. Machacek, E. Anderson, C. Makochekanwa, S. J. Buckman, and J. P. Sullivan, Phys. Rev. A 88, 042715 (2013). 